\title{
Robust stability analysis for uncertain delay systems with output feedback controller
}

\author{
Chwan-Lu Tseng, I-Kong Fong and Juing-Huei Su \\ Department of Electrical Engineering, National Taiwan University, Taipei, Taiwan 10617, ROC
}

Received 13 May 1993

Revised 6 October 1993

\begin{abstract}
A systematic approach is given in this paper for analyzing the robust stability of uncertain time-delay systems controlled by output feedback. By checking the eigenvalues of a Hamiltonian matrix, the stability of nominal systems can be examined first. Then, for the nominally stable uncertain systems with multiple time delays, a new method using structured singular value technique is proposed for finding a set of uncertain parameters within which the systems remain stable. Moreover, an illustrative example is given to show the usefulness of the proposed approach.
\end{abstract}

Keywords: Time-delay system; output feedback; Hamiltonian matrix; robust stability; structured singular value.

\section{Introduction}

For control systems with both time delays and uncertainties, the stability analysis problem is usually complicated. Recently, several robust stability test methods are proposed for such systems with various types of uncertainties $[2,9,12,13,16]$. However, to consider uncertain time-delay systems controlled by output feedback controller, these methods cannot be applied directly because when there are uncertainties in the input and output matrices of the open-loop system, multiplicative terms of uncertainties will appear in the resulting closed-loop system. For the case of cone bounded uncertainties, i.e. uncertainties of which norms are bounded by that of the state vector, Chou et al. [3] extend the results of [16] to deal with systems equipped with dynamical output feedback controller. For the case of structured uncertainties in the system matrix, of which the modulus matrix is bounded by a given constant matrix, a time domain approach is proposed by $\mathrm{Yu}$ et al. [15] to check the robust stability of systems with static output feedback controller. However, when there are linearly dependent parametric uncertainties, the multiplicative terms turn out to be quadratic functions of the uncertain parameters, and these methods still lead to conservative results.

In this paper, the robust stability problem of the case with quadratically coupled parametric uncertainties is investigated by means of the Lyapunov functional approach [7]. For uncertain systems with multiple time delays, we start by deriving a sufficient condition to check whether the uncertain system is nominally stable. If the nominal systems are stable, we proceed to study the derivative of a Lyapunov functional candidate of the uncertain systems. We use a transformation method which is equivalent to the completion of square in the associated Lyapunov derivative and a method based on the structured singular value (henceforth denoted as $\mu$ ) technique $[1,4]$ to find a set of uncertainties within which the derivative is negative definite. Then, an example is given to illustrate the usefulness of the approach.

The notations adopted are as follows. Let $h$ be a positive constant. $x_{t}(\sigma)=x(t+\sigma)$ for $-h \leqslant \sigma \leqslant 0 .\|x\|_{f}=$ $\sup _{-h \leqslant \sigma \leqslant 0}\|x(\sigma)\|$, where $\|\cdot\|$ is the Euclidean norm. $C_{h}$ is the Banach space of continuous vector functions $\eta:[-h, 0] \rightarrow \mathbb{R}^{n}$ with $\|\cdot\|_{f} . \otimes$ refers to the Kronecker product, and $I_{\delta}$ is the identity matrix of dimension $\delta$.

Correspondence to: I.-K. Fong, Department of Electrical Engineering, National Taiwan University, Taipei, Taiwan 10617, ROC. 


\section{Stability analysis of uncertain output feedback systems with multiple time delays}

Consider the uncertain system $\left(\Sigma_{m}\right)$ described by the following functional differential equation and controlled by output feedback

$$
\begin{aligned}
\dot{x}(t)= & \left(A_{0}+\sum_{i=1}^{m} k_{i} A_{i}\right) x(t)+\left(D_{0}+\sum_{i=1}^{m} k_{i} D_{i}\right) x(t-h) \\
& +\sum_{l=1}^{q} \sum_{i=1}^{m} k_{i} F_{i l} x\left(t-h_{l}(t)\right)+\left(B_{0}+\sum_{i=1}^{m} k_{i} B_{i}\right) u(t), \\
y(t)= & \left(C_{0}+\sum_{i=1}^{m} k_{i} C_{i}\right) x(t), \\
u(t)=L y(t), & \\
x(t)= & \phi(t) \quad \forall t \in\left[-h_{\max }, 0\right],
\end{aligned}
$$

where $x \in \mathbb{R}^{n}, u \in \mathbb{R}^{p}$ and $y \in \mathbb{R}^{r}$ are the state, input, and output vectors, respectively, $L \in \mathbb{R}^{p \times r}$ is the output feedback gain matrix, $h>0$ is the constant delay time, $h_{l}(t), l=1, \ldots, q$, are time-varying bounded delay times satisfying $0 \leqslant h_{l}(t) \leqslant h_{l}<\infty$ and $\dot{h}_{l}(t) \leqslant d_{l}<1, \phi(t) \in C_{h_{\max }}, h_{\max }=\max \left\{h, \max _{l} h_{l}\right\}, d_{\max }=\max _{l} d_{l}$, and $k_{i} \in \mathbb{R}, i=1, \ldots, m$, are the time-varying uncertain parameters satisfying $\max _{i}\left|k_{i}\right|<\gamma$. In addition, the matrices $A_{i}, B_{i}, C_{i}, D_{i}$, and $F_{i l}$ of suitable dimensions are the structural influence matrices of $k_{i}$, and are assumed to be known. Thus, the closed-loop system can be written as

$$
\begin{aligned}
\dot{x}(t)= & \left(\bar{A}_{0}+\sum_{i=1}^{m} k_{i} E_{i}+\sum_{i, j=1}^{m} k_{i} k_{j} E_{i j}\right) x(t)+\left(D_{0}+\sum_{i=1}^{m} k_{i} D_{i}\right) x(t-h) \\
& +\sum_{i=1}^{q} \sum_{i=1}^{m} k_{i} F_{i l} x\left(t-h_{l}(t)\right),
\end{aligned}
$$

where $\bar{A}_{0} \triangleq A_{0}+B_{0} L C_{0}, E_{i} \triangleq B_{0} L C_{i}+B_{i} L C_{0}$, and $E_{i j} \triangleq B_{i} L C_{j}$. The objectives of the following discussions are to derive a stability condition for the nominal system, and to find how large $\gamma$ can be if the stability of the closed-loop uncertain system is to be preserved.

Consider the nominal system $\left(\Sigma_{0}\right)$,

$$
\dot{x}(t)=\bar{A}_{0} x(t)+D_{0} x(t-h),
$$

which is the system $\left(\Sigma_{m}\right)$ with $k_{i}=0$ for all $i$. We have the following theorem.

Theorem 1. If $\bar{A}_{0}$ is a stable matrix and for some $\alpha>0$

$$
H=\left[\begin{array}{cc}
\bar{A}_{0} & D_{0} D_{0}^{\mathrm{T}} \\
-(\alpha+1) I_{n} & -\bar{A}_{0}^{\mathrm{T}}
\end{array}\right]
$$

has no eigenvalues on the $\mathrm{j} \omega$-axis, then the system $\Sigma_{0}$ is asymptotically stable.

Proof. From the assumptions and Lemma 4 in [5], it is known that the algebraic Riccati equation (ARE)

$$
\bar{A}_{0}^{\mathrm{T}} P+P \bar{A}_{0}+P D_{0} D_{0}^{\mathrm{T}} P+(\alpha+1) I_{n}=0
$$

has a positive-definite solution $P$ for some $\alpha>0$. Consider the Lyapunov functional candidate

$$
V\left(t, x_{t}\right)=x^{\mathrm{T}}(t) P x(t)+\int_{t-h}^{t} x^{\mathrm{T}}(\sigma) x(\sigma) \mathrm{d} \sigma .
$$


Then the Lyapunov derivative $\dot{V}\left(t, x_{t}\right)$ is

$$
\begin{aligned}
& x^{\mathrm{T}}(t)\left(\bar{A}_{0}^{\mathrm{T}} P+P \bar{A}_{0}+I_{n}\right) x(t)+2 x(t)^{\mathrm{T}} P D_{0} x(t-h)-x^{\mathrm{T}}(t-h) x(t-h) \\
& =\left[x^{\mathrm{T}}(t) x^{\mathrm{T}}(t-h)\right]\left[\begin{array}{cc}
\bar{A}_{0}^{\mathrm{T}} P+P \bar{A}_{0}+I_{n} & P D_{0} \\
D_{0}^{\mathrm{T}} P & -I_{n}
\end{array}\right]\left[\begin{array}{c}
x(t) \\
x(t-h)
\end{array}\right] \\
& =\left[x^{\mathrm{T}}(t) x^{\mathrm{T}}(t-h)\right]\left[\begin{array}{cc}
I_{n} & -P D_{0} \\
0 & I_{n}
\end{array}\right] \\
& \times\left[\begin{array}{cc}
\bar{A}_{0}^{\mathrm{T}} P+P \bar{A}_{0}+P D_{0} D_{0}^{\mathrm{T}} P+I_{n} & 0 \\
0 & -I_{n}
\end{array}\right]\left[\begin{array}{cc}
I_{n} & 0 \\
-D_{0}^{\mathrm{T}} P & I_{n}
\end{array}\right]\left[\begin{array}{c}
x(t) \\
x(t-h)
\end{array}\right] .
\end{aligned}
$$

Since $P$ is the solution of ARE (3), we have

$$
\dot{V}\left(t, x_{t}\right) \leqslant-\alpha\|x(t)\|^{2} .
$$

Thus, we conclude this theorem by using the Lyapunov stability theorem [7, p. 105].

From Theorem 1, it is known that the stability of the nominal system $\Sigma_{0}$ can be guaranteed if ARE (3) has a positive-definite solution. However, in the presence of uncertainties and time-varying delays, it is intuitively clear that the stability condition would be more stringent. To proceed, we assume the nominal system satisfies the assumptions of Theorem 1 for $\alpha>1$. Thus the nominal system is asymptotically stable, and ARE (3) has a positive-definite solution $P$ for some $\alpha>1$. This is equivalent to assuming that the ARE

$$
\bar{A}_{0}^{\mathrm{T}} P+P \bar{A}_{0}+P D_{0} D_{0}^{\mathrm{T}} P+(\beta+2) I_{n}=0, \quad \beta>0
$$

has a positive-definite solution $P$. In other words, we make a little stronger assumption to cope with the additional terms in the system dynamics. We then turn to our objective: the robust stability of the uncertain system.

For the uncertain system $\left(\Sigma_{m}\right)$, consider the Lyapunov functional candidate

$$
V\left(t, x_{t}\right)=x^{\mathrm{T}}(t) P x(t)+\int_{t-h}^{t} x^{\mathrm{T}}(\sigma) x(\sigma) \mathrm{d} \sigma+\frac{1}{q} \sum_{l=1}^{q} \int_{t-h_{l}(t)}^{t} x^{\mathrm{T}}(\sigma) x(\sigma) \mathrm{d} \sigma .
$$

Then, with the satisfaction ARE (5), it is easy to show that the Lyapunov derivative can be written as

$$
\begin{aligned}
\dot{V}\left(t, x_{t}\right)= & x^{\mathrm{T}}(t)\left[P \bar{A}_{0}+\bar{A}_{0}^{\mathrm{T}} P+2 I_{n}+\sum_{i=1}^{m} k_{i}\left(P E_{i}+E_{i}^{\mathrm{T}} P\right)+\sum_{i, j=1}^{m} k_{i} k_{j}\left(P E_{i j}+E_{i j}^{\mathrm{T}} P\right)\right] x(t) \\
& +2 x^{\mathrm{T}}(t) P\left(D_{0}+\sum_{i=1}^{m} k_{i} D_{i}\right) x(t-h)+2 x^{\mathrm{T}}(t) P \sum_{l=1}^{q} \sum_{i=1}^{m} k_{i} F_{i l} x\left(t-h_{l}(t)\right) \\
& -x^{\mathrm{T}}(t-h) x(t-h)-\frac{1}{q} \sum_{l=1}^{q}\left[1-\dot{h}_{l}(t)\right] x^{\mathrm{T}}\left(t-h_{l}(t)\right) x\left(t-h_{l}(t)\right) \\
\leqslant & x^{\mathrm{T}}(t) T Q T^{\mathrm{T}} x(t),
\end{aligned}
$$

where $x(t)=\left[x^{\mathrm{T}}(t) x^{\mathrm{T}}(t-h) x^{\mathrm{T}}\left(t-h_{1}(t)\right) \cdots x^{\mathrm{T}}\left(t-h_{\mathrm{q}}(t)\right)\right]^{\mathrm{T}}$,

$$
\boldsymbol{T}=\left[\begin{array}{ccccc}
I_{n} & -P\left(D_{0}+\sum_{i=1}^{m} k_{i} D_{i}\right) & -P \sum_{i=1}^{m} k_{i} F_{i l} & \cdots & -P \sum_{i=1}^{m} k_{i} F_{i q} \\
0 & I_{n} & 0 & \cdots & 0 \\
0 & 0 & I_{n} & \ddots & \vdots \\
\vdots & & & 0 & I_{n}
\end{array}\right]
$$


and

$$
Q=\left[\begin{array}{ccccc}
S(k) & 0 & 0 & \cdots & 0 \\
0 & -I_{n} & 0 & & \vdots \\
\vdots & & -\frac{1-d_{\max }}{q} I_{n} & & \\
0 & 0 & \ldots & 0 & -\frac{1-d_{\max }}{q} I_{n}
\end{array}\right],
$$

$$
\begin{aligned}
S(k)= & -\beta I_{n}+\sum_{i=1}^{m} k_{i}\left[P E_{i}+E_{i}^{\mathrm{T}} P+P\left(D_{0} D_{i}^{\mathrm{T}}+D_{i} D_{0}^{\mathrm{T}}\right) P\right] \\
& +\sum_{i, j=1}^{m} k_{i} k_{j}\left[P E_{i j}+E_{i j}^{\mathrm{T}} P+\frac{1}{2} P\left(D_{i} D_{j}^{\mathrm{T}}+D_{j} D_{i}^{\mathrm{T}}\right) P+\frac{q}{2\left(1-d_{\max }\right)} \sum_{l=1}^{q} P\left(F_{i l} F_{j l}^{\mathrm{T}}+F_{j l} F_{i l}^{\mathrm{T}}\right) P\right] \\
= & -\beta\left[I_{n}+\sum_{i=1}^{m} k_{i} \bar{E}_{i}+\sum_{i, j=1}^{m} k_{i} k_{j} \bar{E}_{i j}\right],
\end{aligned}
$$

with

$$
\begin{aligned}
& \bar{E}_{i} \triangleq-\frac{1}{\beta}\left[P E_{i}+E_{i}^{\mathrm{T}} P+P\left(D_{0} D_{i}^{\mathrm{T}}+D_{i} D_{0}^{\mathrm{T}}\right) P\right], \\
& \bar{E}_{i j} \triangleq-\frac{1}{\beta}\left[P E_{i j}+E_{i j}^{\mathrm{T}} P+\frac{1}{2} P\left(D_{i} D_{j}^{\mathrm{T}}+D_{j} D_{i}^{\mathrm{T}}\right) P\right]-\frac{q}{2 \beta\left(1-d_{\max }\right.} \sum_{l=1}^{q} P\left(F_{i l} F_{j l}^{\mathrm{T}}+F_{j l} F_{i l}^{\mathrm{T}}\right) P,
\end{aligned}
$$

and

$$
k \triangleq\left[k_{1} \cdots k_{m}\right]
$$

Note that basically the transformation procedure in relation (7) is completing the square and transferring the Lyapunov derivative into a quadratic form. Consequently, it is readily seen that $\dot{V}\left(t, x_{t}\right)$ is negative-definite if $S(k)$ is negative-definite for all $\left|k_{i}\right|<\gamma, i=1, \ldots, m$. This is true if and only if

$$
\begin{aligned}
\operatorname{det}\left(I_{n}+\sum_{i=1}^{m} k_{i} \bar{E}_{i}+\sum_{i, j=1}^{m} k_{i} k_{j} \bar{E}_{i j}\right) & =\operatorname{det}\left[I_{n}+\left(k \otimes I_{n}\right)^{\mathrm{T}} \bar{E}_{e}+\left(k \otimes I_{n}\right)^{\mathrm{T}} \bar{E}_{e e}\left(k \otimes I_{n}\right)\right] \\
& =\operatorname{det}\left\{I_{(m+1) n}+\left[\begin{array}{cc}
\left(k \otimes I_{n}\right)^{\mathrm{T}} & 0 \\
0 & k \otimes I_{n}
\end{array}\right]\left[\begin{array}{cc}
\bar{E}_{e} & \bar{E}_{e e} \\
-I_{n} & 0
\end{array}\right]\right\} \\
& \neq 0
\end{aligned}
$$

where

$$
\bar{E}_{e}=\left[\begin{array}{lllll}
\bar{E}_{1}^{\mathrm{T}} & \bar{E}_{2}^{\mathrm{T}} & \cdots & \bar{E}_{m}^{\mathrm{T}}
\end{array}\right]^{\mathrm{T}} \text { and } \bar{E}_{e e}=\left[\begin{array}{cccc}
\bar{E}_{11} & \bar{E}_{12} & \cdots & \bar{E}_{1 m} \\
\bar{E}_{21} & \bar{E}_{22} & & \bar{E}_{2 m} \\
\vdots & & \cdots & \vdots \\
\bar{E}_{m 1} & \bar{E}_{m 2} & \cdots & \bar{E}_{m m}
\end{array}\right] \text {, }
$$

since $S(k)=-\beta I_{n}$ is negative-definite when $k=0$, and the eigenvalues of $S(k)$ cannot become positive when $\left|k_{i}\right|$ 's vary from 0 to $\gamma$. It is easy to check that inequality $(10)$ holds if and only if

$$
\operatorname{det}\left\{I_{(m+1) n}+\sum_{i=1}^{m} k_{i} U_{i} \overline{\boldsymbol{E}}\right\} \neq 0,
$$


where $U_{i}=\left(e_{1} e_{i}^{\mathrm{T}}+e_{i+1} e_{m+1}^{\mathrm{T}}\right) \otimes I_{n}, e_{j}$ is the $j$ th column of $I_{m+1}$, and

$$
\bar{E}=\left[\begin{array}{cc}
\bar{E}_{e} & \bar{E}_{e e} \\
-I_{n} & 0
\end{array}\right] \text {. }
$$

Thus, we can obtain the following theorem.

Theorem 2. The uncertain delay system $\left(\Sigma_{m}\right)$ is asymptotically stable if

$$
\gamma<\mu^{-1}(M),
$$

where

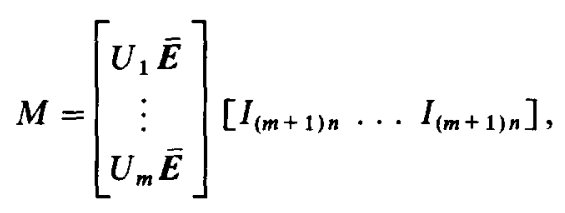

and $\mu(\cdot)$ denotes the structured singular value [4] with respect to the set of real uncertainty matrices of the form $\bar{\Delta}=\operatorname{diag}\left[k_{1} I_{(m+1) n}, \ldots, k_{m} I_{(m+1) n}\right]$.

Proof. Using the definition of the structured singular value, we know that inequality (10) holds if and only if inequality (12) holds. Thus, $S(k)$ is negative-definite with the satisfaction of inequality (12). Hence, there exist an $\varepsilon>0$ such that $\dot{V}\left(t, x_{t}\right) \leqslant-\varepsilon\|x(t)\|^{2}$. This concludes the proof.

Note that if all $F_{i l}$ 's are absent, it is possible to use the Lyapunov functional (4) and the assumption of Theorem 1 instead of $(6)$ and that of Theorem 2 to show the robust asymptotic stability of $\left(\Sigma_{m}\right)$. This reduces some conservativeness because sometimes the solution of ARE (3) exists while that of ARE (5) does not.

The use of $\mu$-analysis technique to treat the negative-definiteness preservation problem of a perturbed matrix is also adopted in [14] to handle the robust stability analysis problems of nondelayed uncertain systems. In fact such approach can be generalized to process the so-called rank preservation problem, and applied to many problems in robust control theory. Although the numerical computation of $\mu$ with respect to real uncertainties is a difficult problem $[6,10]$, the well-developed $\mu$-analysis software [1] for complex uncertainties can be used to provide an upper bound. As we shall see in the subsequent example, in spite of the possible existence of conservativeness which is not caused by the proposed approach, but by the adopted computation algorithm, the application results are quite satisfactory.

\section{An example}

Consider the uncertain system $\left(\Sigma_{m}\right)$ with the following data: $m=2, q=1, d_{\max }=0.5$,

$$
\begin{aligned}
& A_{0}=\left[\begin{array}{rr}
-1 & 5 \\
1 & -1
\end{array}\right], \quad B_{0}=\left[\begin{array}{l}
1 \\
1
\end{array}\right], \quad C_{0}^{\mathrm{T}}=\left[\begin{array}{c}
0.5 \\
1
\end{array}\right], \quad D_{0}=\left[\begin{array}{ll}
0.5 & 0 \\
0.75 & 1
\end{array}\right], \\
& A_{1}=\left[\begin{array}{ll}
0 & 1 \\
1 & 0
\end{array}\right], \quad B_{1}=\left[\begin{array}{l}
0 \\
1
\end{array}\right], \quad C_{2}^{\mathrm{T}}=\left[\begin{array}{l}
0 \\
1
\end{array}\right], \quad D_{1}=\left[\begin{array}{ll}
0 & 0 \\
0 & 1
\end{array}\right], \quad F_{11}=\left[\begin{array}{ll}
0 & 1 \\
0 & 0
\end{array}\right],
\end{aligned}
$$

$A_{2}, B_{2}, C_{1}, D_{2}$ and $F_{21}$ are zero matrices of suitable dimensions, and the output feedback gain $L=-4$. Thus, we have

$$
\bar{A}_{0}=\left[\begin{array}{rr}
-3 & 1 \\
-1 & -5
\end{array}\right],
$$


which is a stable matrix. By using Theorem 1, it is found that ARE (3) has a positive-definite solution

$$
P=\left[\begin{array}{ll}
0.3324 & 0.0195 \\
0.0195 & 0.2112
\end{array}\right]
$$

at $\alpha=1$. Hence, the nominal system is asymptotically stable. This agrees with the result in [8]. In addition, using Theorem 2 and the $\mu$-analysis package [1], we know that ARE (5) has a positive-definite solution

$$
P=\left[\begin{array}{ll}
0.5020 & 0.0325 \\
0.0325 & 0.3237
\end{array}\right]
$$

at $\beta=1$, and get $\mu^{-1}(M)=0.1600$. As a result, we know that the system $\left(\Sigma_{m}\right)$ remains asymptotically stable provided $\max _{i}\left|k_{i}\right|<0.1600$, even if $k_{i}$ 's are time-varying. Figure 1 shows the region of negative-definite $S(k)$ in the parameter plane for $\beta=1$ (so the stability region guaranteed by the specific Lyapunov functional), which is the region to the right of the dotted line, the boundary on which $\operatorname{det}[S(k)]=0$. The dotted line is obtained by letting $k_{2}=\rho k_{1}$ with a constant $\rho$, solving the equation $\operatorname{det}[S(k)]=0$ for the only variable $k_{1}$, and repeating the procedure for various values of $\rho$. The square in the center is the boundary of the derived uncertainty range, of which the lower left corner almost touches the boundary. This shows that a nonconservative result is obtained in this example, although $\mu$-analysis algorithm for complex uncertainties is applied. Moreover, by using Theorem 2.1 of [11], it is known that if there exists an $\beta^{*}$ such that ARE (5) has a positive-definite solution, then it has positive-definite solutions for all $\beta \in\left(0, \beta^{*}\right]$. Since $\mu^{-1}(M)$ depends on $M$, which in turns depends on $P$ and hence $\beta$, better results may be found if a simple one-dimensional search over $\beta$ is carried out. In this example, it is found that the largest $\beta^{*} \in[10.4,10.5)$. Thus the plot of $\mu^{-1}[M(\beta)]$ is depicted in Figure 2 for $0.1 \leqslant \beta \leqslant 10.4$ with increment 0.5 . Indeed, a better bound 0.3367 is found around $\beta=6.1$.

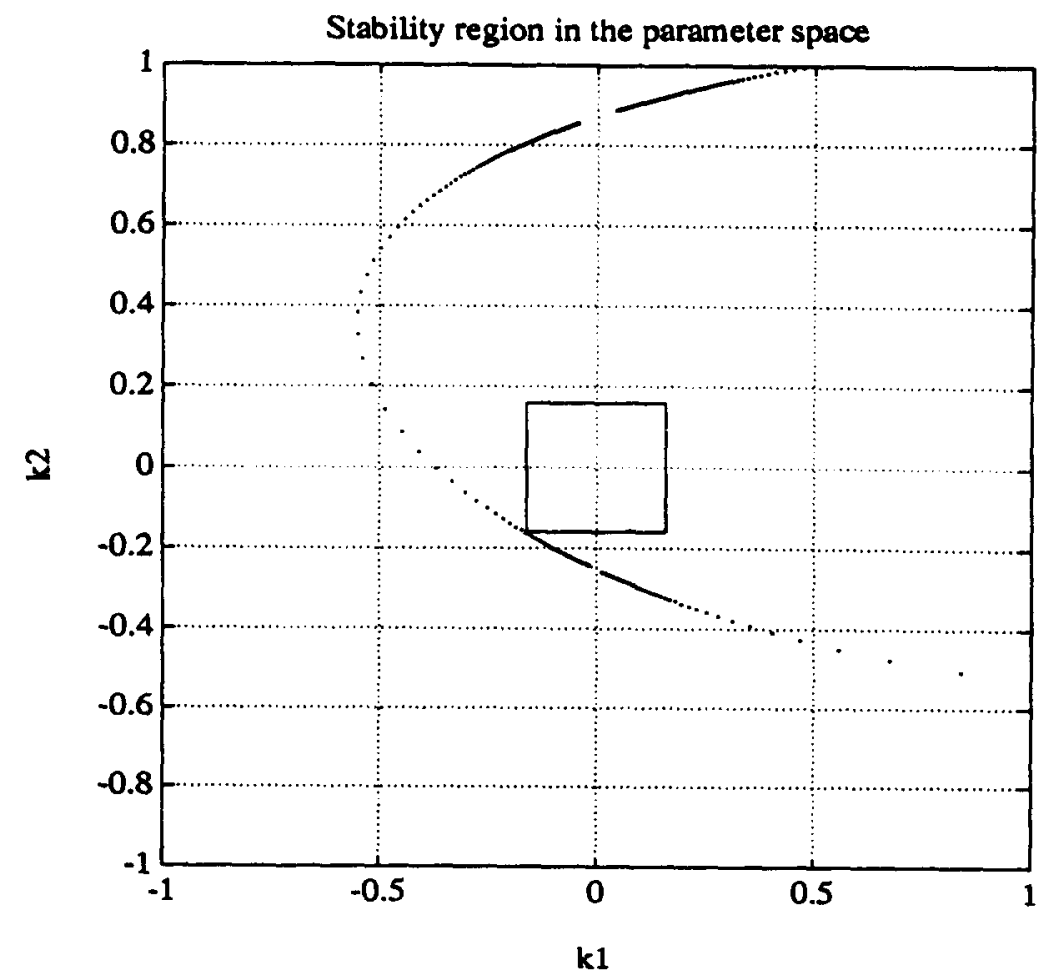

Fig. 1. The region of negative definite $S(k)$ for $\beta=1$. 


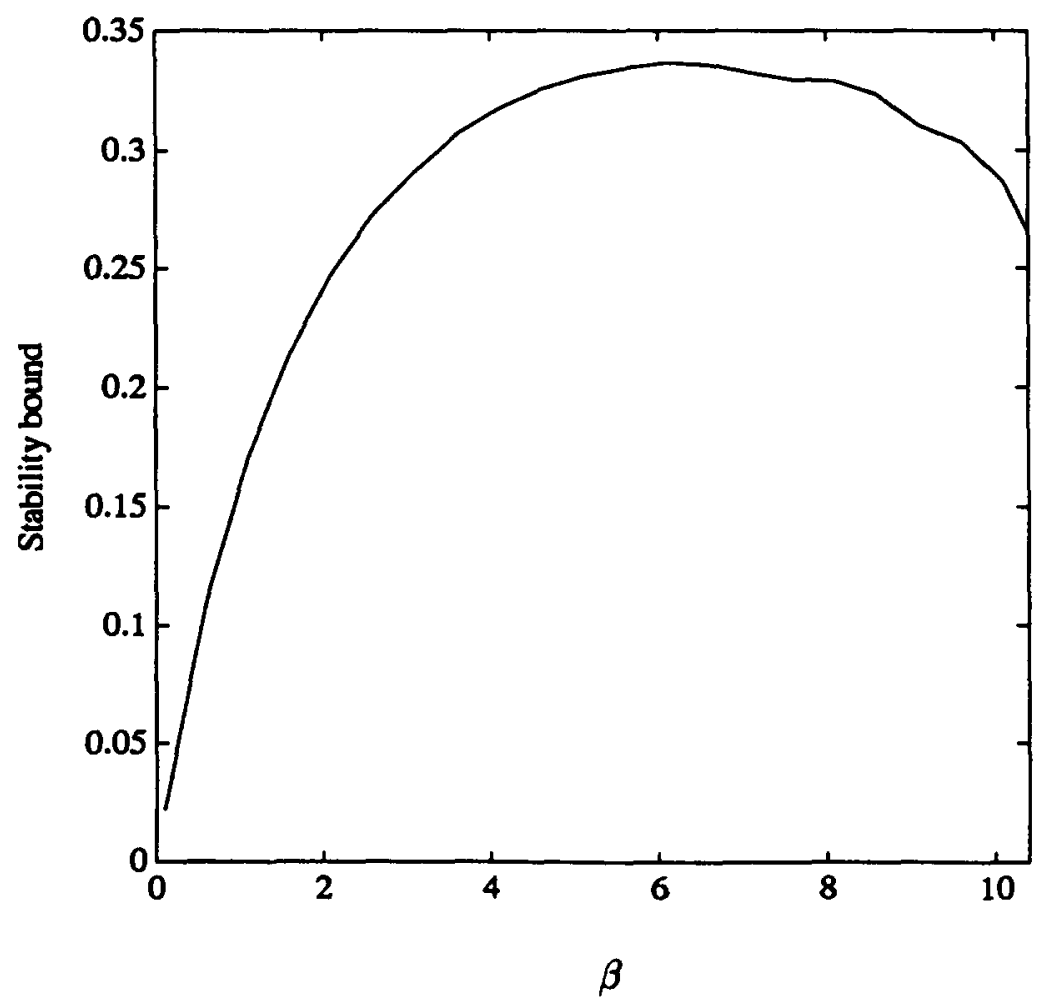

Fig. 2. The plot of $\mu^{-1}[M(\beta)]$.

\section{Conclusion}

In this paper, we propose a new approach to deal with the robust stability problem of the uncertain time-delay systems controlled by static output feedback. To check whether the uncertain systems are nominally stable, we prove that it suffices to check locations of the eigenvalues of an associated Hamiltonian matrix. For the uncertain systems which are nominally stable and possibly have multiple noncommensurate time-varying delays, a transform method is given to transfer the Lyapunov derivative of the system into a quadratic form. Then it is shown that with the $\mu$-analysis technique we can find a set of uncertainties within which the uncertain time-delay systems keep asymptotic stability. A numerical example is provided to illustrate the use of the proposed approach, and a one-dimensional search is suggested for improving the final results.

\section{References}

[1] G.J. Balas, J.C. Doyle, K. Glover, A Packard and R. Smith, $\mu$-Analysis and Synthesis Toolbox - User's Guide (Musyn Inc. and The Math Works Inc., Natick, MA, 1991).

[2] E. Cheres, Z.J. Palmor and S. Gutman, Quantitative measures of robustness for systems including delayed perturbations, IEEE Trans. Automat. Control 34 (1989) 1203-1204.

[3] J.H. Chou, I.R. Horng and B.S. Chen, Dynamical feedback compensator for uncertain time-delay systems containing saturating actuator, Internat. J. Control 49 (1989) 961-968.

[4] J.C. Doyle, Analysis of feedback systems with structured uncertainties, Proc. IEE-D 129 (1982) 242-250.

[5] J.C. Doyle, K. Glover, P.P. Khargonekar and B.A. Francis, State-space solutions to standard $H_{2}$ and $H_{\infty}$ control problem, IEEE Trans. Automat. Control 34 (1989) 831-846. 
[6] M. Elgersma, J. Freudenberg and B. Morton, Polynomial methods for the structured singular value with real parameters, in: Proc. 31st IEEE Conf. on Decision and Control, Tucson, Arizona (1992) 237-242.

[7] J. Hale, Theory of Functional Differential Equations (Springer, New York, 1977).

[8] A. Hmamed, Further results on the delay-independent asymptotic stability of linear systems, Internat. J. Systems Sci. 22 (1991) 1127-1132.

[9] A. Hmamed, Further results on the robust stability of uncertain time-delay systems, Internat. J. Systems Sci. 22 (1991) 605-614.

[10] M.P. Newlin and P.M. Young, Mixed $\mu$ problems and branch and bound techniques, in: Proc. 31st IEEE Conf. on Decision and Control, Tucson, Arizona (1992) 3175-3180.

[11] I.R. Petersen, Some new results on algebraic Riccati equations arising in linear quadratic differential games and the stabilization of uncertain linear systems, Systems Control Lett. 10 (1992) 341-348.

[12] S. Phoojaruenchanachai and K. Furuta, Memoryless stabilization of uncertain linear systems including time-varying state delays, IEEE Trans. Automat. Control 37 (1992) 1022-1026.

[13] T.J. Su and C.G. Huang, Robust stability of delay dependence for linear uncertain systems, IEEE Trans. Automat. Control 37 (1992) 1656-1659.

[14] C.L. Tseng, I.K. Fong and J.H. Su, Stability analysis of systems with structured time-varying uncertainties using structured singular value, presented in the 3rd SIAM Conf. on Linear Algebra in Signals, Systems and Control, Seattle, Washington, 1993.

[15] W. Yu, K.M. Sobel and E.Y. Shapiro, A time domain approach to the robustness of time delay systems, in: Proc. 3Ist IEEE Conf. on Decision and Control, Tucson, Arizona (1992) 3726-3727.

[16] S.S. Wang, B.S. Chen and T.P. Lin, Robust stability of uncertain time-delay systems, Internat. J. Control 46 (1987) $963-976$. 